\title{
Embeddings of local automata
}

\author{
Marie-Pierre Béal, Sylvain Lombardy and Dominique Perrin
}

September 23, 2009

\begin{abstract}
We prove that any local irreducible automaton is contained in a complete one. The proof uses Nasu's masking lemma. We use the same technique to give a new proof that any weakly deterministic irreducible automaton is contained in a complete one.
\end{abstract}

\section{Contents}

1 Introduction 1

2 Equivalences of automata 2

$\begin{array}{lll}3 & \text { Local automata } & 9\end{array}$

4 Weakly deterministic automata $\quad 16$

\section{Introduction}

The problem of embedding a finite automaton into one having specified properties is an old one in automata theory (see [11] for example). We will consider here the problem of embedding an automaton into a complete one for several families of automata including local and weakly deterministic automata.

In symbolic dynamics, the problem of embedding a shift into another shift has been studied extensively. A necessary and sufficient condition for the strict embedding of shifts of finite type has been given by Krieger (Krieger's Embedding Theorem [7]).

The problem of embedding an unambiguous irreducible automaton into a complete one has been solved in [1] using a construction due to Ehrenfeucht and Rozenberg [5]. This result has a formulation in terms of codes. Indeed, the stabilizer of a state in an unambiguous automaton is a free submonoid. Thus the above embedding implies that any rational code is contained in a maximal one (the Ehrenfeucht-Rozenberg Theorem).

A local automaton is by definition such that a bounded information about the past and the future is enough to determine the present state. Formally there 
are integers $\lambda, \rho$ such that for any pair of paths $p \stackrel{u}{\rightarrow} q \stackrel{v}{\rightarrow} r$ and $p^{\prime} \stackrel{u}{\rightarrow} q^{\prime} \stackrel{v}{\rightarrow} r^{\prime}$ having the same label with $|u|=\lambda$ and $|v|=\rho$ one has $q=q^{\prime}$. A subautomaton of a local automaton is obviously still local but conversely it is not clear how one can add transitions or states to a local automaton until it is complete, in the sense that any word on the underlying alphabet is the label of a path.

The problem of completing local automata has already been considered in [8], where a method is given which allows one to complete a local deterministic automaton when it is possible. We prove here that any local automaton is always contained in a complete one. The stabilizer of a state in a local automaton is the star of a locally parsable code (also called codes with finite synchronization delay). The previous result gives an alternative proof of a result of Bruyère according to which any locally parsable rational code is included in a maximal one [4], see also [3].

Our proof relies on a result known as Nasu's Masking Lemma [10]. This result deals with the embedding of a shift of finite type into another one and gives a complement to Krieger's Embedding Theorem. Given a graph $G$, the edge shift on $G$ is the set $X_{G}$ of biinfinite paths in $G$. The result states that if $X_{G}$ can be embedded in $X_{H}$, then there is a graph $K$ such that $X_{K}$ is isomorphic to $X_{H}$ and $G$ is contained in $K$. We use this result in the particular case where $X_{H}$ is the full shift but the same proof can be used to obtain a more general result with an arbitrary subshift instead of the full shift.

We also use Nasu's masking lemma to give a new proof of a result of [1] concerning weakly deterministic automata, which are such that the knowledge of the starting state and of a prefix of length $d$ of the label of a path determines its first edge. These automata are also called right-closing in symbolic dynamics. We give a new proof using Nasu's lemma that any $d$-weakly deterministic automaton is contained in a complete one. This result can be reformulated in terms of codes. The basis of the stabilizer of a state in a weakly deterministic automaton is, by definition, a weakly prefix code (also called codes with finite deciphering delay).

We have written this paper in a self-contained form and in particular we adapt the proof of Nasu's lemma to our notation.

A first version of this paper was presented at the IEEE International Symposium on Information Theory [2].

\section{Equivalences of automata}

We denote by $\mathcal{A}=(Q, E)$ a finite automaton on the alphabet $A$ with $Q$ as set of states and $E \subset Q \times A \times Q$ as set of edges. We consider all states as both initial and terminal. The automaton is said to be complete (with respect to the alphabet $A$ ) if for any word $w \in A^{*}$ there exists a path labelled $w$. It is said to be unambiguous if for any $p, q \in Q$ and $w$ in $A^{*}$ there is at most one path from $p$ to $q$ labelled $w$. It is said to be irreducible if for any $p, q \in Q$ there is at least one word $w$ such that there is a path from $p$ to $q$ labelled $w$.

An automaton is said to be essential if any state has at least one incom- 
ing edge and at least one outgoing edge. Clearly an irreducible automaton is essential.

An automaton $\mathcal{A}=(P, E)$ on the alphabet $A$ is a subautomaton of an automaton $\mathcal{B}=(Q, F)$ on the alphabet $A$, if $P \subset Q$ and $E \subseteq F \cap(P \times A \times P)$.

An automaton is deterministic if for each state $p$ and each letter $a$ there is at most one edge labelled $a$ going out of $p$. We use the notation $p \cdot a=q$ to express the fact that there is an edge $p \stackrel{a}{\rightarrow} q$ in a deterministic automaton. It can be shown that an irreducible deterministic automaton is complete if and only if for each state $p$ and letter $a$ there is exactly one edge labelled $a$ going out of $p$.

The transition matrix of an automaton $\mathcal{A}=(Q, E)$ is the $Q \times Q$ matrix with elements in the set $\mathfrak{P}(A)$ of subsets of $A$ defined for $p, q \in Q$ by

$$
M_{p, q}=\{a \in A \mid \text { there is an edge }(p, a, q) \text { in } E\} .
$$

The elements of $M$ can be considered as elements of the semiring $\mathfrak{P}\left(A^{*}\right)$ of subsets of $A^{*}$, where 0 is the empty set and 1 is the set containing the empty word. Such matrices can therefore be multiplied.

Let $\mathcal{A}=(P, E)$ and $\mathcal{B}=(Q, F)$ be two automata on the alphabet $A$. Let $M$ be the transition matrix of $\mathcal{A}$ and let $N$ be the transition matrix of $\mathcal{B}$. We say that $\mathcal{A}$ and $\mathcal{B}$ are elementary equivalent if there exist a $(P \times Q)$-matrix $R$ and a $(Q \times P)$-matrix $S$ both with elements in $\mathfrak{P}(A) \cup\{1\}$, where 1 is the empty word, such that $M=R S$ and $N=S R$. We also say that $\mathcal{A}$ is $(R, S)$-elementary equivalent to $\mathcal{B}$.

This notion is classical in symbolic dynamics. It is usually formulated for subshifts of finite type (see [7] p. 225). Our definition is a particular case of the notion of symbolic elementary equivalence for sofic shifts introduced in [9].

For a $P \times Q$-matrix $R$ with elements in $\mathfrak{P}(A) \cup\{1\}$, we say that a triple $(p, a, q) \in P \times(A \cup\{1\}) \times Q$ is an $R$-edge (resp. an $S$-edge) when $a \in R_{p, q}$ (resp. $\left.a \in S_{p, q}\right)$. Thus when $\mathcal{A}$ is $(R, S)$-equivalent to $\mathcal{B}$, each edge of $\mathcal{A}$ is the sequence of an $R$-edge and a consecutive $S$-edge, and each edge of $\mathcal{B}$ is a sequence of an $S$-edge and a consecutive $R$-edge. The $R$-edges and $S$-edges can be considered as edges of a bipartite graph called the auxiliary graph of the equivalence (it is called a bipartite code in [9] and [7, p. 355]).

Example 1 Let $\mathcal{A}$ and $\mathcal{B}$ be the automata represented on Figure 1.
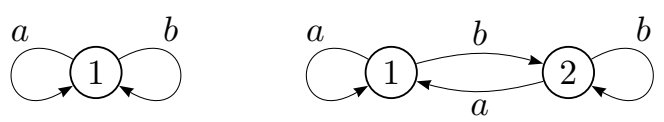

Figure 1: Two elementary equivalent automata $\mathcal{A}$ (on the left) and $\mathcal{B}$ (on the right).

The transition matrices $M$ and $N$ of $\mathcal{A}$ and $\mathcal{B}$ are

$$
M=[a+b], \quad N=\left[\begin{array}{ll}
a & b \\
a & b
\end{array}\right] .
$$


Let

$$
R=\left[\begin{array}{ll}
a & b
\end{array}\right], \quad S=\left[\begin{array}{l}
1 \\
1
\end{array}\right] .
$$

Then $M=R S$ and $N=S R$. Thus $\mathcal{A}$ and $\mathcal{B}$ are elementary equivalent. The auxiliary graph is shown in Figure 2 (with continuous lines).

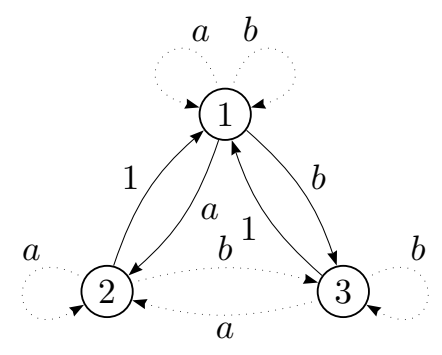

Figure 2: The auxiliary graph (with continuous edges).

Let $\mathcal{A}=(Q, E)$ be an automaton. The input of a state $q \in Q$ is the set of pairs $(p, a)$ such that one has $p \stackrel{a}{\rightarrow} q$. Its output is the set of pairs $(a, r)$ such that one has $q \stackrel{a}{\rightarrow} r$.

An input merge equivalence is an equivalence on the set $Q$ of states of $\mathcal{A}$ such that for any pair $p, p^{\prime}$ of equivalent states, any letter $a$ and any state $q$, one has

(i) $p \stackrel{a}{\rightarrow} q$ if and only if $p^{\prime} \stackrel{a}{\rightarrow} q$,

(ii) $q \stackrel{a}{\rightarrow} p, q \stackrel{a}{\rightarrow} p^{\prime}$ implies $p=p^{\prime}$.

Thus, in such an equivalence, two equivalent and distinct states have the same output and disjoint inputs.

The quotient of $\mathcal{A}=(Q, E)$ by such an equivalence is the automaton $\overline{\mathcal{A}}=$ $(\bar{Q}, \bar{E})$ with states the set $\bar{Q}$ of equivalence classes, and edges the induced edges on the classes, i.e. $(p, a, q) \in E$ for some $p, q \in Q$ implies that $(\bar{p}, a, \bar{q})$ is in $\bar{E}$.

Thus, in the quotient the output of a class of states is the common output of its elements and its input is the union, or merge, of the inputs of its elements (whence the name of an input merge equivalence). We say that $\overline{\mathcal{A}}$ is obtained from $\mathcal{A}$ by an input merge. We also say that $\mathcal{A}$ is obtained from $\overline{\mathcal{A}}$ by an input split .

Note that if $\mathcal{A}$ is an unambiguous essential automaton, there is a largest input merge equivalence. It is defined by $q \equiv q^{\prime}$ if $q$ and $q^{\prime}$ have the same output. Indeed, since $\mathcal{A}$ is essential, if $q \equiv q^{\prime}$, they have the same non-empty output. Since it is unambiguous, they have disjoint inputs.

Example 2 Let $\mathcal{A}, \mathcal{B}$ be the automata of Example 1. The automaton $\mathcal{A}$ is obtained from $\mathcal{B}$ by an input merge. 
An output merge equivalence on the set of states of an automaton $\mathcal{A}$ is defined symmetrically. It is an equivalence on the set $Q$ of states such that equivalent and distinct states have the same input and disjoint outputs. The quotient $\overline{\mathcal{A}}$ is defined in the same way by merging the outputs of the elements of a class. We say that $\overline{\mathcal{A}}$ is obtained from $\mathcal{A}$ by an output merge. We also say that $\mathcal{A}$ is obtained from $\overline{\mathcal{A}}$ by an output split .

Observe that the quotient of an automaton $\mathcal{A}$ by an input or an output merge equivalence is unambiguous if and only if $\mathcal{A}$ is unambiguous.

The notions of input and output merge equivalence are classical in symbolic dynamics. They are usually formulated for subshifts of finite type (see [7, p. 225]). The extension of the definitions to sofic shifts is due to Nasu [9]; see also $[6]$. $[7])$.

The following result is an element of William's Classification Theorem (see

Proposition 1 If the automaton $\mathcal{A}$ is obtained from the automaton $\mathcal{B}$ by an input (or output) merge, then $\mathcal{A}$ and $\mathcal{B}$ are elementary equivalent.

Proof We treat the case of an input merge. Let $\mathcal{B}=(Q, E)$ and $\mathcal{A}=(\bar{Q}, \bar{E})$. Let $M$ and $N$ be the transition matrices of $\mathcal{A}$ and $\mathcal{B}$. Let $R$ be the $\bar{Q} \times Q$-matrix defined for $p, q \in Q$ by

$$
R_{\bar{p}, q}=\{a \in A \mid \text { there is an edge }(p, a, q) \text { in } E\}
$$

(note that $R$ is well-defined because of the definition of an input merge). Let $S$ be the $Q \times \bar{Q}$-matrix defined by

$$
S_{p, \bar{q}}=\left\{\begin{array}{cc}
1 & \text { if } \bar{p}=\bar{q} \\
0 & \text { otherwise }
\end{array}\right.
$$

Then $M=R S$ and $N=S R$. Thus $\mathcal{A}$ and $\mathcal{B}$ are elementary equivalent.

Proposition 2 Let $\mathcal{A}$ and $\mathcal{B}$ be two irreducible $(R, S)$-elementary equivalent automata. Then, either $R$ has elements in $\mathfrak{P}(A)$ and $S$ in $\{0,1\}$ or conversely.

Proof Set $\mathcal{A}=(P, E)$ and $\mathcal{B}=(Q, F)$. Suppose that there are $p \in P, a \in A$, and $q \in Q$ such that $a \in R_{p q}$. Let $p^{\prime} \in P$ and $q^{\prime} \in Q$. We denote $s=S_{q^{\prime} p^{\prime}}$. Let us show that, if $s \neq 0$ then $s=1$. Since $\mathcal{B}$ is irreducible, there is path $q \stackrel{u}{\rightarrow} q^{\prime}$ labelled by $u=a_{1} \ldots a_{n}$. We have $a_{i}=s_{i} r_{i}$, for $1 \leq i \leq n$, with $r_{i} \in R_{p_{i} q_{i}}$, and $s_{i} \in S_{q_{i-1} p_{i}}$, and thus the path

$$
p \stackrel{a}{\rightarrow} q_{0} \stackrel{s_{1}}{\rightarrow} p_{1} \stackrel{r_{1}}{\rightarrow} q_{1} \stackrel{s_{2}}{\rightarrow} \cdots \stackrel{s_{n}}{\rightarrow} p_{n} \stackrel{r_{n}}{\rightarrow} q_{n} \stackrel{s}{\rightarrow} p^{\prime}
$$

with $q_{0}=q$ and $q_{n}=q^{\prime}$. Since $a s_{1}$ is the label of an edge in $E, s_{1}=1$. Since $s_{1} r_{1}$ is the label of an edge in $F, r_{1} \in A$ and so on. Finally $r_{n} \in A$ and thus $s=1$.

This shows that if one entry of $R$ contains an element of $A$ then all the entries of $S$ are 0 or 1. Furthermore, if all the entries of $S$ are 0 or 1, then all 
non-null entries of $R$ are in $\mathfrak{P}(A)$. The proof of the symmetrical case is similar.

We denote by $L(\mathcal{A})$ the set of words labelling a finite path in $\mathcal{A}$. Two automata $\mathcal{A}$ and $\mathcal{B}$ are said to be equivalent if $L(\mathcal{A})=L(\mathcal{B})$.

We say that two automata $\mathcal{A}$ and $\mathcal{B}$ are strongly equivalent if there is a sequence $\mathcal{A}_{0}, \mathcal{A}_{1}, \ldots, \mathcal{A}_{n}$ of automata such that $\mathcal{A}_{0}=\mathcal{A}, \mathcal{A}_{n}=\mathcal{B}$ and $\mathcal{A}_{i}$ is elementary equivalent to $\mathcal{A}_{i+1}$ for $0 \leq i \leq n-1$.

Proposition 3 Two strongly equivalent essential automata are equivalent.

Proof Let $\mathcal{A}=(P, E)$ and $\mathcal{B}=(Q, F)$ be $(R, S)$-elementary equivalent automata on the alphabet $A$. Let $w=a_{1} \ldots a_{n} \in L(\mathcal{A})$. Since $\mathcal{A}$ is essential, there is a path

$$
p_{-1} \stackrel{a_{0}}{\rightarrow} p_{0} \stackrel{a_{1}}{\rightarrow} p_{1} \stackrel{a_{2}}{\rightarrow} \cdots p_{n-1} \stackrel{a_{n}}{\rightarrow} p_{n} \stackrel{a_{n+1}}{\longrightarrow} p_{n+1},
$$

with $a_{0}, a_{n+1} \in A$. For each $i=0, \ldots, n+1$, we have $a_{i}=r_{i} s_{i}$ with $r_{i} \in R_{p_{i-1} q_{i}}$ and $s_{i} \in S_{q_{i} p_{i}}$, and thus a path

$$
p_{-1} \stackrel{r_{0}}{\rightarrow} q_{0} \stackrel{s_{0}}{\rightarrow} p_{0} \stackrel{r_{1}}{\rightarrow} q_{1} \stackrel{s_{1}}{\rightarrow} p_{1} \cdots p_{n-1} \stackrel{r_{n}}{\rightarrow} q_{n} \stackrel{s_{n}}{\rightarrow} p_{n} \stackrel{r_{n+1}}{\rightarrow} q_{n+1} \stackrel{s_{n+1}}{\rightarrow} p_{n+1}
$$

using alternately entries of $R$ and $S$. Then either $r_{0}=a_{0}, \ldots, r_{n+1}=a_{n+1}$ and $s_{0}=\cdots=s_{n+1}=1$ or $r_{0}=\cdots=r_{n+1}=1$ and $s_{0}=a_{0}, \ldots, s_{n+1}=a_{n+1}$. We consider the first case, the other one being symmetrical. Since $S R$ is the transition matrix of $\mathcal{B}$, we have in $\mathcal{B}$ a path

$$
q_{0} \stackrel{a_{1}}{\rightarrow} q_{1} \stackrel{a_{2}}{\rightarrow} \cdots \stackrel{a_{n}}{\rightarrow} q_{n} \stackrel{a_{n+1}}{\rightarrow} q_{n+1} .
$$

This shows that $w a_{n+1} \in L(\mathcal{B})$ and thus $w \in L(\mathcal{B})$.

We will now describe a construction due to Nasu (see [7, p. 354], Lemma 10.2.3). It associates to automata $\mathcal{A}_{1}, \mathcal{A}_{2}, \mathcal{B}_{1}$ such that $\mathcal{A}_{1}$ is elementary equivalent to $\mathcal{A}_{2}$ and $\mathcal{A}_{1}$ is a subautomaton of $\mathcal{B}_{1}$, an automaton $\mathcal{B}_{2}$ elementary equivalent to $\mathcal{B}_{1}$ and such that $\mathcal{A}_{2}$ is a subautomaton of $\mathcal{B}_{2}$. The automaton $\mathcal{B}_{2}$ is called the Nasu embedding of $\mathcal{A}_{2}$ with respect to $\mathcal{A}_{1}, \mathcal{B}_{1}$.

Set $\mathcal{A}_{1}=\left(P_{1}, E_{1}\right), \mathcal{A}_{2}=\left(P_{2}, E_{2}\right)$ and $\mathcal{B}_{1}=\left(Q_{1}, F_{1}\right)$. Let $R, S$ be matrices such that $R S$ and $S R$ are respectively the transition matrices of $\mathcal{A}_{1}$ and $\mathcal{A}_{2}$. Let $Q_{2}=P_{2} \cup\left(F_{1} \backslash E_{1}\right)$. We define a $Q_{1} \times Q_{2}$-matrix $\widehat{R}$ and a $Q_{2} \times Q_{1}$-matrix $\widehat{S}$ as follows.

Suppose first that $R$ has 0,1 entries and $S$ entries in $\mathfrak{P}(A)$. Then, for $q_{1} \in Q_{1}$ and $q_{2} \in Q_{2}$, we define

$$
\widehat{R}_{q_{1}, q_{2}}= \begin{cases}R_{q_{1}, q_{2}} & \text { if } q_{1} \in P_{1}, q_{2} \in P_{2} \\ 1 & \text { if } q_{2} \in F_{1} \backslash E_{1} \text { and } o\left(q_{2}\right)=q_{1} \\ 0 & \text { otherwise }\end{cases}
$$

and

$$
\widehat{S}_{q_{2}, q_{1}}=S_{q_{2}, q_{1}} \cup\left\{l\left(q_{2}\right) \mid q_{2} \in F_{1} \backslash E_{1}, e\left(q_{2}\right)=q_{1}\right\},
$$


where $o\left(q_{2}\right)$ denotes the origin of $q_{2}, l\left(q_{2}\right)$ its label, and $e\left(q_{2}\right)$ its end.

An illustration of this construction is given in Example 3.

In the case where $R$ has entries in $\mathfrak{P}(A)$ and $S$ has 0,1 entries, we define

$$
\widehat{R}_{q_{1}, q_{2}}=R_{q_{1}, q_{2}} \cup\left\{a \in A \mid q_{2} \in F_{1} \backslash E_{1}, o\left(q_{2}\right)=q_{1}, l\left(q_{2}\right)=a\right\}
$$

and

$$
\widehat{S}_{q_{2}, q_{1}}= \begin{cases}S_{q_{2}, q_{1}} & \text { if } q_{1} \in P_{1}, q_{2} \in P_{2} \\ 1 & \text { if } q_{2} \in F_{1} \backslash E_{1} \text { and } e\left(q_{2}\right)=q_{1} \\ 0 & \text { otherwise }\end{cases}
$$

It is clear that in both cases, $\widehat{R} \widehat{S}$ is the transition matrix of $\mathcal{B}_{1}$. The automaton $\mathcal{B}_{2}=\left(Q_{2}, F_{2}\right)$ is defined by its transition matrix which is $\widehat{S} \widehat{R}$. To sum up, we have the following statement.

Proposition 4 Let $\mathcal{A}_{1}, \mathcal{A}_{2}, \mathcal{B}_{1}$ be automata such that $\mathcal{A}_{1}$ is a subautomaton of $\mathcal{B}_{1}$ and $\mathcal{A}_{1}$ is elementary equivalent to $\mathcal{A}_{2}$. Let $\mathcal{B}_{2}$ be the Nasu embedding of $\mathcal{A}_{2}$ with respect to $\mathcal{A}_{1}, \mathcal{B}_{1}$. Then $\mathcal{A}_{2}$ is a subautomaton of $\mathcal{B}_{2}$ and $\mathcal{B}_{1}$ is elementary equivalent to $\mathcal{B}_{2}$. Moreover, if $\mathcal{A}_{2}$ and $\mathcal{B}_{1}$ are irreducible (resp. essential), then $\mathcal{B}_{2}$ is irreducible (resp. essential).

Example 3 Figure 3 represents the Nasu embedding $\mathcal{B}_{2}$ of $\mathcal{A}_{2}$ with respect to $\mathcal{A}_{1}, \mathcal{B}_{1}$. The automata $\mathcal{A}_{1}$ and $\mathcal{A}_{2}$ are the elementary equivalent automata of Example 1. If $M_{i}$ (resp. $N_{i}$ ) denotes the transition matrix of the automaton $\mathcal{A}_{i}$

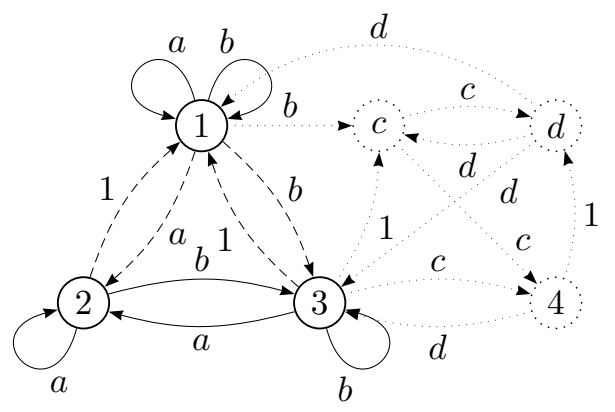

Figure 3: The Nasu embedding $\mathcal{B}_{2}$ of $\mathcal{A}_{2}$ with respect to $\mathcal{A}_{1}, \mathcal{B}_{1}$. The automata $\mathcal{A}_{1}=\left(P_{1}, E_{1}\right)$ with $P_{1}=\{2,3\}$ and $\mathcal{A}_{2}=\left(P_{2}, E_{2}\right)$ with $P_{2}=\{1\}$ are represented with continuous edges. We have $\mathcal{B}_{1}=\left(Q_{1}, F_{1}\right)$ with $Q_{1}=\{2,3,4\}$ and $\mathcal{B}_{2}=\left(Q_{2}, F_{2}\right)$ with $Q_{1}=\{1, c, d\}$. The automaton $\mathcal{B}_{2}$ has two additional states $c, d$ corresponding to the additional edges $(3, c, 4)$ and $(4, d, 3)$ of $\mathcal{B}_{1}$. The transitions defined by $R$ and $S$ are represented with dashed edges. Additional states and edges are represented with dots.

(resp. $\left.\mathcal{B}_{i}\right)$ of Figure 3, we have

$$
M_{1}=\left[\begin{array}{ll}
a & b \\
a & b
\end{array}\right]=R S=\left[\begin{array}{l}
1 \\
1
\end{array}\right]\left[\begin{array}{ll}
a & b
\end{array}\right], M_{2}=[a+b]=S R .
$$




$$
\begin{gathered}
N_{1}=\left[\begin{array}{lll}
a & b & 0 \\
a & b & c \\
0 & d & 0
\end{array}\right]=\widehat{R} \widehat{S}=\left[\begin{array}{lll}
1 & 0 & 0 \\
1 & 1 & 0 \\
0 & 0 & 1
\end{array}\right]\left[\begin{array}{lll}
a & b & 0 \\
0 & 0 & c \\
0 & d & 0
\end{array}\right], \\
N_{2}=\left[\begin{array}{ccc}
a+b & b & 0 \\
0 & 0 & c \\
d & d & 0
\end{array}\right]=\widehat{S} \widehat{R}=\left[\begin{array}{lll}
a & b & 0 \\
0 & 0 & c \\
0 & d & 0
\end{array}\right]\left[\begin{array}{lll}
1 & 0 & 0 \\
1 & 1 & 0 \\
0 & 0 & 1
\end{array}\right] .
\end{gathered}
$$

The following result will be used in Section 4 .

Proposition 5 Let $\mathcal{A}_{1}, \mathcal{A}_{2}, \mathcal{A}_{3}, \mathcal{B}_{3}$ be essential automata such that $\mathcal{A}_{1}$ is an input merge of $\mathcal{A}_{2}, \mathcal{A}_{3}$ is an output merge of $\mathcal{A}_{2}$ and $\mathcal{A}_{3}$ is a subautomaton of $\mathcal{B}_{3}$.

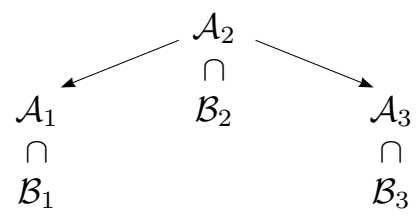

Let $\mathcal{B}_{2}$ be the Nasu extension of $\mathcal{A}_{2}$ with respect to $\mathcal{A}_{3}, \mathcal{B}_{3}$. Then $\mathcal{A}_{1}$ is a subautomaton of the maximal input merge $\mathcal{B}_{1}$ of $\mathcal{B}_{2}$.

Proof Set $\mathcal{A}_{i}=\left(P_{i}, E_{i}\right)$ for $i=1,2,3$ and let $\mathcal{B}_{i}=\left(Q_{i}, F_{i}\right)$ for $i=2,3$. Let $M_{i}$ be the transition matrix of $\mathcal{A}_{i}$ for $i=1,2,3$. Set $M_{2}=S R, M_{3}=R S$ and let $\widehat{R}, \widehat{S}$ be the matrices corresponding to the Nasu extension of $\mathcal{A}_{2}$. We have $Q_{2}=P_{2} \cup\left(F_{3} \backslash E_{3}\right)$ and for $q_{2} \in Q_{2}, q_{3} \in Q_{3}$,

$$
\widehat{S}_{q_{2}, q_{3}}=S_{q_{2}, q_{3}} \cup\left\{l\left(q_{2}\right) \mid q_{2} \in F_{3} \backslash E_{3}, e\left(q_{2}\right)=q_{3}\right\}
$$

and

$$
\widehat{R}_{q_{3}, q_{2}}= \begin{cases}R_{q_{2}, q_{3}} & \text { if } q_{2} \in P_{2}, q_{3} \in P_{3} \\ 1 & \text { if } q_{2} \in F_{3} \backslash E_{3} \text { and } o\left(q_{2}\right)=q_{3} \\ 0 & \text { otherwise }\end{cases}
$$

Let $p_{2}, p_{2}^{\prime} \in P_{2}$ be two states of $\mathcal{A}_{2}$ that have the same output. Let us show that they also have the same output in $\mathcal{B}_{2}$. For this, suppose that $\left(p_{2}, a, r\right)$ is an edge of $\mathcal{B}_{2}$ with $r \in F_{3} \backslash E_{3}$. Since the transition matrix of $\mathcal{B}_{2}$ is $\widehat{S} \widehat{R}$, there is a state $q \in Q_{3}$ such that $a \in \widehat{S}_{p_{2} q}$ and $\widehat{R}_{q r}=1$. It follows from the definition of $\widehat{S}$ that $q$ is in $P_{3}$ (indeed if $q \in Q_{3} \backslash P_{3}$, then $p_{2} \in F_{3} \backslash E_{3}$, a contradiction). Since $\mathcal{A}_{3}$ is obtained from $\mathcal{A}_{2}$ by an output merge, there is a $p \in P_{2}$ such that $R_{q, p}=1$ and thus an edge $\left(p_{2}, a, q\right)$ in $E_{2}$. Since $p_{2}, p_{2}^{\prime}$ have the same input in $\mathcal{A}_{2}$, we have also an edge $\left(p_{2}^{\prime}, a, q\right)$ in $E_{2}$. Thus there is an edge $\left(p_{2}^{\prime}, a, r\right)$ in $F_{2}$, which proves the assertion.

This shows that the automaton $\mathcal{B}_{1}$ obtained from $\mathcal{B}_{2}$ by merging the states with the same output, has $\mathcal{A}_{1}$ as a subautomaton. 


\section{Local automata}

An automaton is said to be $(\lambda, \rho)$-local if for all pairs of paths $p \stackrel{u}{\rightarrow} q \stackrel{v}{\rightarrow} r$ and $p^{\prime} \stackrel{u}{\rightarrow} q^{\prime} \stackrel{v}{\rightarrow} r^{\prime}$ with $|u|=\lambda$ and $|v|=\rho$, one has $q=q^{\prime}$. The automaton is said to be local if it is $(\lambda, \rho)$-local for some $\lambda, \rho \geq 0$. An automaton which has a single state is $(0,0)$-local.

Thus in a local automaton, one can recover a biinfinite path from its label using a sliding window of fixed size.

As a particular case of a local automaton, an edge automaton is such that all edges have distinct labels. An edge automaton is both $(0,1)$ and $(1,0)$-local.

A word $w$ is said to be a constant for an automaton $\mathcal{A}$ if for $p, p^{\prime}, q, q^{\prime} \in P$ such that $p \stackrel{w}{\rightarrow} q$ and $p^{\prime} \stackrel{w}{\rightarrow} q^{\prime}$ one has also $p \stackrel{w}{\rightarrow} q^{\prime}$ and $p^{\prime} \stackrel{w}{\rightarrow} q$. The empty word is a constant only when the automaton has a single state.

Observe that when the automaton is deterministic, a constant is a word $w$ such that the set $\operatorname{Im}(w)=\{q \in P \mid p \stackrel{w}{\rightarrow} q$ for some $p \in P\}$ has at most one element.

The following result from [8] gives a necessary a property of irreducible complete deterministic local automata. This property can be used to show that some local deterministic automata are not contained in a complete irreducible one (see Example 6).

Proposition 6 Let $\mathcal{A}$ be is an irreducible complete deterministic local automaton on the alphabet $A$ with more than one state. Then

$$
\bigcap_{a \in A} \operatorname{Im}(a)=\emptyset
$$

Proof Suppose that $q \in \bigcap_{a \in A} \operatorname{Im}(a)$. Let $w$ be a word of maximal length which is not a constant. Then for each letter $a \in A$, the word $a w$ is a constant and thus $\operatorname{Im}(a w)$ has only one element. Thus for any state $p$ we have $p \cdot w=q \cdot w$. Indeed, since $\mathcal{A}$ is essential, there is a letter $a$ such that $p \in \operatorname{Im}(a)$. Then $p, q \in \operatorname{Im}(a)$, which implies $p \cdot w=q \cdot w$. This contradicts the fact that $w$ is not a constant.

Example 4 The automaton of Figure 4 is $(1,1)$-local. All nonempty words but $a$ are constants.

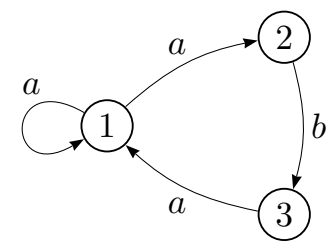

Figure 4: A (1,1)-local automaton. 
Note that if $\mathcal{A}$ is a $(\lambda, \rho)$-local automaton, then an input split of $\mathcal{A}$ is a $(\lambda+$ $1, \rho)$-local automaton, and an output split of $\mathcal{A}$ is a $(\lambda, \rho+1)$-local automaton.

Proposition 7 An irreducible automaton is local if and only if it is unambiguous and there exists an integer $n$ such that any word of length $n$ is a constant.

Proof Suppose first that $\mathcal{A}$ is $(\lambda, \rho)$-local. Let $c, c^{\prime}$ be two paths from $p$ to $q$ labelled $w$. Since $\mathcal{A}$ is irreducible, there exist states $r, s$ and paths $r \stackrel{u}{\rightarrow} p, q \stackrel{v}{\rightarrow} s$ with $|u|=\lambda$ and $|v|=\rho$. Since $\mathcal{A}$ is $(\lambda, \rho)$-local, the paths $c, c^{\prime}$ coincide. Thus $\mathcal{A}$ is unambiguous. Suppose next that $p \stackrel{w}{\rightarrow} q$ and $p^{\prime} \stackrel{w}{\rightarrow} q^{\prime}$ with $|w|=\lambda+\rho$. Let $w=u v$ with $|u|=\lambda$ and $|v|=\rho$. Let $r, r^{\prime}$ be such that $p \stackrel{u}{\rightarrow} r \stackrel{v}{\rightarrow} q$ and $p^{\prime} \stackrel{u}{\rightarrow} r^{\prime} \stackrel{v}{\rightarrow} q^{\prime}$. Since $\mathcal{A}$ is $(\lambda, \rho)$-local, we have $r=r^{\prime}$. This implies that we also have paths $p \stackrel{w}{\rightarrow} q^{\prime}$ and $p^{\prime} \stackrel{w}{\rightarrow} q$. Thus the condition of the statement is true with $n=\lambda+\rho$.

Conversely, let us assume that any word of length $n$ is a constant and that $\mathcal{A}$ is unambiguous. Let us show that $\mathcal{A}$ is $(n, n)$-local. Suppose that $p \stackrel{u}{\rightarrow} q \stackrel{v}{\rightarrow} r$ and $p^{\prime} \stackrel{u}{\rightarrow} q^{\prime} \stackrel{v}{\rightarrow} r^{\prime}$ with $|u|=|v|=n$. Then, since $u$ and $v$ are constants, we also have $p \stackrel{u}{\rightarrow} q^{\prime} \stackrel{v}{\rightarrow} r$. Since $\mathcal{A}$ is unambiguous, this forces $q=q^{\prime}$, whence the conclusion.

The least integer $n$ such that any word of length $n$ is a constant is called the order of the automaton. Note that a $(\lambda, \rho)$-local automaton has order at most $\lambda+\rho$. It can however be strictly less than $\lambda+\rho$. An unambiguous automaton with order $n$ is $(n, n)$-local.

Let $\lambda, \rho \geq 0$. The free $(\lambda, \rho)$-local automaton is the automaton with set of states consisting of pairs $(u, v)$ of words, with $u$ of length $\lambda$ and $v$ of length $\rho$, and edges the triples $\left((u, v), a,\left(u^{\prime}, v^{\prime}\right)\right)$ such that there are letters $b, c$ with $u v c=b u^{\prime} v^{\prime}$ and $a$ is the first letter of $v c$ (and also the last letter of $\left.b u^{\prime}\right)$. It is clear that this automaton is $(\lambda, \rho)$-local.

The free $(n, 0)$-local automaton is usually known as the de Bruijn automaton of order $n$.

Lemma 1 Let $n$ be a nonnegative integer such that $\lambda, \rho \leq n$, the free $(\lambda, \rho)$-local automaton has order $n$.

Proof Let $\mathcal{F}_{(\lambda, \rho)}$ be the free $(\lambda, \rho)$-local automaton. Let $(u, v) \stackrel{w}{\rightarrow}(x, y)$ and $\left(u^{\prime}, v^{\prime}\right) \stackrel{w}{\rightarrow}\left(x^{\prime}, y^{\prime}\right)$ be two paths labelled $w$ of length $n$ in $\mathcal{F}_{(\lambda, \rho)}$, with $u, u^{\prime}, x, x^{\prime}$ of length $\lambda$ and $v, v^{\prime}, y, y^{\prime}$ of length $\rho$. Since $\lambda, \rho \leq n, v, v^{\prime}$ are prefixes of $w$ and $x, x^{\prime}$ are suffixes of $w$. Hence $v=v^{\prime}$ and $x=x^{\prime}$ and there are paths $(u, v) \stackrel{w}{\rightarrow}\left(x, y^{\prime}\right)$ and $\left(u^{\prime}, v\right) \stackrel{w}{\rightarrow}(x, y)$ in $\mathcal{F}_{(\lambda, \rho)}$.

Example 5 The free $(1,1)$-local automaton on the alphabet $\{a, b\}$ is represented on Figure 5. The label of an edge going out of a state is its second letter.

The following result shows that any $(\lambda, \rho)$-local automaton is strongly equivalent to a subautomaton of the free $(\lambda, \rho)$-local automaton. 


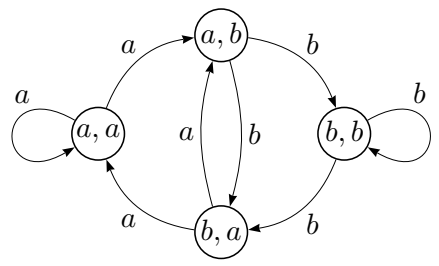

Figure 5: The free $(1,1)$-local automaton. It has order 1.

Proposition 8 If $\mathcal{A}$ is $(\lambda, \rho)$-local automaton, there is a sequence $\mathcal{A}_{0}, \mathcal{A}_{1}, \ldots, \mathcal{A}_{\lambda+\rho}$ of $(\lambda, \rho)$-local automata such that

(i) $\mathcal{A}_{0}=\mathcal{A}$.

(ii) For $i=0, \ldots, \lambda-1, \mathcal{A}_{i}$ is obtained from $\mathcal{A}_{i+1}$ by an input merge.

(iii) For $i=\lambda, \ldots, \lambda+\rho-1, \mathcal{A}_{i}$ is obtained from $\mathcal{A}_{i+1}$ by an output merge.

(iv) $\mathcal{A}_{\lambda+\rho}$ is a subautomaton of the free $(\lambda, \rho)$-local automaton.

Proof Let $\mathcal{A}=(P, E)$. We define for $1 \leq i \leq \lambda, \mathcal{A}_{i}=\left(P_{i}, E_{i}\right)$ where $P_{i}$ is the set of pairs $(u, q)$ with $u \in A^{i}$ and $q \in Q$ such that there is a path labelled $u$ leading to $q$ in $\mathcal{A}$. There is an edge labelled $a$ from $(u, p)$ to $(v, q)$ in $\mathcal{A}_{i}$ if $u=b u^{\prime}$, $v=u^{\prime} a$ for $b \in A$ and $(p, a, q) \in E$. For $j=1, \ldots, \rho$, let $\mathcal{A}_{\lambda+j}=\left(P_{\lambda+j}, E_{\lambda+j}\right)$ where $P_{\lambda+j}$ is the set of triples $(u, p, v) \in A^{\lambda} \times P \times A^{j}$ such that there is a path labelled $u$ leading to $p$ and a path labelled $v$ leaving $p$ in $\mathcal{A}$. There is an edge labelled $a$ from $(u, p, v)$ to $(w, q, t)$ if and only if $u=b u^{\prime}, w=u^{\prime} a, v=a v^{\prime}$, $t=v^{\prime} c$ and $(p, a, q) \in E$.

For $0 \leq i \leq \lambda-1$, the equivalence $\theta_{i+1}$ on $P_{i+1}$ defined by $(u, p) \equiv\left(u^{\prime}, p^{\prime}\right)$ if $p=p^{\prime}$ and $u, u^{\prime}$ differ at most by the first letter is an input merge. Similarly, for $\lambda \leq i \leq \lambda+\rho-1$, the equivalence $\theta_{i+1}$ on $P_{i+1}$ defined by $(u, p, v) \equiv\left(u^{\prime}, p^{\prime}, v^{\prime}\right)$ if $u=u^{\prime}, p=p^{\prime}$ and $v, v^{\prime}$ differ at most by their last letter, is an output merge. This shows that conditions (ii) and (iii) are satisfied.

Finally, since $\mathcal{A}$ is $(\lambda, \rho)$-local, in a state $(u, p, v)$ of $A_{\lambda+\rho}$, the state $p$ is determined by $(u, v)$. Thus condition (iv) is also satisfied.

Observe that if $\mathcal{A}$ is moreover supposed to be irreducible, then all the automata $\mathcal{A}_{i}$ constructed as above are also irreducible.

We will prove the following result.

Theorem 2 Any local local irreducible automaton is a subautomaton of a complete one with the same order.

The proof uses Nasu embeddings. We will deduce Theorem 2 from the following more precise statement. 
Proposition 9 Any irreducible $(\lambda, \rho)$-local automaton is a subautomaton of a complete irreducible local automaton of order $\max (\lambda, \rho)$.

Note that it is not true that any irreducible $(\lambda, \rho)$-local automaton is a subautomaton of a complete irreducible $(\lambda, \rho)$-local automaton (see Example 6).

The proof of Proposition 9 uses the following statement.

Proposition 10 Let $\mathcal{A}_{1}$ and $\mathcal{A}_{2}$ be automata such that $\mathcal{A}_{2}$ is obtained from $\mathcal{A}_{1}$ by input (or output) merge. If $\mathcal{A}_{1}$ is a subautomaton of a local automaton $\mathcal{B}_{1}$, then the Nasu embedding of $\mathcal{A}_{2}$ with respect to $\mathcal{A}_{1}$ and $\mathcal{B}_{1}$, is a local automaton with the same order as $\mathcal{B}_{1}$.

Proof We treat the case of an input merge. The case of an output merge is symmetrical. Let $\mathcal{B}_{1}=\left(Q_{1}, F_{1}\right)$ be a local automaton containing $\mathcal{A}_{1}=\left(P_{1}, E_{1}\right)$ as a subautomaton. Assume that $\mathcal{A}_{2}=\left(P_{2}, E_{2}\right)$ is obtained from $\mathcal{A}_{1}$ by input merge. Let $F=F_{1} \backslash E_{1}, Q_{2}=P_{2} \cup F$ and let $\mathcal{B}_{2}=\left(Q_{2}, F_{2}\right)$ be the Nasu embedding of $\mathcal{A}_{2}$ with respect to $\mathcal{A}_{1}, \mathcal{B}_{1}$.

Let $R, S$ be the matrices defined as in the proof of Proposition 1. The transition matrix of $\mathcal{A}_{1}$ is equal to $R S$ while the transition matrix of $\mathcal{A}_{2}$ is equal to $S R$. We have $a \in S_{\bar{p}, q}$ if $(p, a, q) \in E_{1}$ and $R_{p, \bar{p}}=1$. The additional $\widehat{S}$-edges are the triples $(f, a, q)$ such that $f=(p, a, q)$ is in $F$. The additional $\widehat{R}$-edges are the triples $(p, 1, f)$ such that $f$ begins with $p$. An example of this construction is described in Figure 3.

Let $\pi$ be the map defined on the set $\mathcal{P}$ of paths of $\mathcal{B}_{1}$ of length at least 2 onto the set of nonempty paths of $\mathcal{B}_{2}$ as follows. If $\left(e_{0}, e_{1}, \ldots, e_{n}\right) \in \mathcal{P}$ with $e_{i}=\left(q_{i}, a_{i}, q_{i+1}\right)$, we define $\pi\left(e_{0}, e_{1}, \ldots, e_{n}\right)=\left(g_{0}, g_{1}, \ldots, g_{n-1}\right)$ with $g_{i}=$ $\left(p_{i}, a_{i}, p_{i+1}\right)$ defined by $p_{i}=\bar{q}_{i}$ if $e_{i} \in E_{1}$ and $p_{i}=e_{i}$ if $e_{i} \in F$.

We claim that $\pi$ is a surjective map from the set $\mathcal{P}$ onto the set of nonempty paths of $\mathcal{B}_{2}$. We have to verify that each $g_{i}$ is an edge of $\mathcal{B}_{2}$. We distinguish four cases.

(i) If $p_{i}, p_{i+1} \in P_{2}$. Then $g_{i}$ is an edge of $\mathcal{A}_{2}$ and thus of $\mathcal{B}_{2}$. It is the concatenation of an $S$-edge and an $R$-edge.

(ii) If $p_{i} \in P_{2}$ and $p_{i+1} \in F$. Then $p_{i}=\bar{q}_{i}$ and $p_{i+1}=e_{i+1}$. Thus $g_{i}$ is the concatenation of an $S$-edge and an $(\widehat{R}-R)$-edge.

(iii) If $p_{i}, p_{i+1}$ are in $F$, then $p_{i}=e_{i}$ and $p_{i+1}=e_{i+1}$. Thus $g_{i}=\left(p_{i}, a_{i}, p_{i+1}\right)$ is the concatenation of an $(\widehat{S}-S)$-edge and an $(\widehat{R}-R)$-edge.

(iv) If $p_{i} \in F$ and $p_{i+1} \in P_{2}$ then $p_{i}=e_{i}$. Thus $g_{i}$ is the concatenation of an $(\widehat{S}-S)$-edge and an $R$-edge.

Thus $\pi$ is well defined. Moreover, one can verify that for any nonempty path $c$ in $\mathcal{B}_{2}$ there exists $c^{\prime} \in \mathcal{P}$ such that $\pi\left(c^{\prime}\right)=c$. Thus $\pi$ is surjective.

Let $n$ be the order of $\mathcal{B}_{1}$. Let $c=p \stackrel{w}{\rightarrow} q$ and $c^{\prime}=p^{\prime} \stackrel{w}{\rightarrow} q^{\prime}$ be two paths in $\mathcal{B}_{2}$ with $|w|=n$. Then there exist paths $d=r \stackrel{w}{\rightarrow} s \stackrel{a}{\rightarrow} t$ and $d^{\prime}=r^{\prime} \stackrel{w}{\rightarrow} s^{\prime} \stackrel{a^{\prime}}{\rightarrow} t^{\prime}$ in 
$\mathcal{B}_{1}$, with $a, a^{\prime} \in A$, such that $\pi(d)=c$ and $\pi\left(d^{\prime}\right)=c^{\prime}$. Since $\mathcal{B}_{1}$ has order $n, w$ is a constant for $\mathcal{B}_{1}$. Thus we have also paths $e=r \stackrel{w}{\rightarrow} s^{\prime} \stackrel{a^{\prime}}{\rightarrow} t^{\prime}$ and $e^{\prime}=r^{\prime} \stackrel{w}{\rightarrow} s \stackrel{a}{\rightarrow} t$ in $\mathcal{B}_{1}$. It is easy to verify that $\pi(e)$ is a path from $p$ to $q^{\prime}$ and $\pi\left(e^{\prime}\right)$ a path from $p^{\prime}$ to $q$. Thus $w$ is a constant for $\mathcal{B}_{2}$.

Proof of Proposition 9. Let $\mathcal{A}$ be an irreducible $(\lambda, \rho)$-local automaton. By Proposition 8 there is a sequence $\mathcal{A}_{0}, \mathcal{A}_{1}, \ldots, \mathcal{A}_{\lambda+\rho}$ of automata such that $\mathcal{A}=$ $\mathcal{A}_{0}, \mathcal{A}_{\lambda+\rho}$ is a subautomaton of the free $(\lambda, \rho)$-local automaton and each $\mathcal{A}_{i}$ is a merge of $A_{i+1}$. By Lemma 1 , the free $(\lambda, \rho)$-local automaton has order $n=\max (\lambda, \rho)$. Since $\mathcal{A}_{\lambda+\rho}$ is included in the free $(\lambda, \rho)$-local automaton, we may build using repeatedly Proposition 10 a sequence $\mathcal{B}_{\lambda+\rho}, \ldots, \mathcal{B}_{1}, \mathcal{B}_{0}$ of complete local irreducible automata with order $n$ such that $\mathcal{A}_{i}$ is contained in $\mathcal{B}_{i}$ for $i=\lambda+\rho, \ldots, 0$. Thus $\mathcal{B}=\mathcal{B}_{0}$ is a complete local automaton with order $n$ containing $\mathcal{A}$. Since $\mathcal{A}$ is irreducible, all $\mathcal{A}_{i}$ and $\mathcal{B}_{i}$ are irreducible.

Example 6 Let $\mathcal{A}=\mathcal{A}_{0}$ be the deterministic automaton represented on the left of Figure 6. It is $(3,0)$-local and has order 3. Indeed, the action of $a, b$ on the set of states represented on the right of Figure 6 shows that any word of length 3 has an image of cardinality at most 1 .
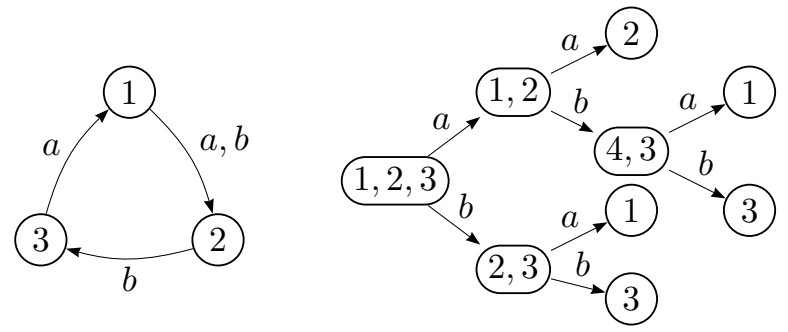

Figure 6: A local automaton $\mathcal{A}$ and the action on subsets.

Note that $\mathcal{A}$ cannot be completed in a local deterministic automaton by Proposition 6. Indeed, we have $2 \in \operatorname{Im}(a) \cap \operatorname{Im}(b)$.

We have represented on Figure 7 a split $\mathcal{A}_{1}$ of $\mathcal{A}$, obtained by an input split of state 2 in two states 5 and 7 . This automaton can easily be completed as indicated on Figure 7.

The matrices $R, S$ such that the transition matrix of $\mathcal{A}_{1}$ is $R S$ and the transition matrix of $\mathcal{A}$ is $S R$ are

$$
R=\left[\begin{array}{lll}
1 & 0 & 0 \\
0 & 1 & 0 \\
0 & 0 & 1 \\
0 & 1 & 0
\end{array}\right], \quad S=\left[\begin{array}{llll}
0 & a & 0 & b \\
0 & 0 & b & 0 \\
a & 0 & 0 & 0
\end{array}\right]
$$

The auxiliary graph is shown on Figure 8. The final result is shown on Figure 9. It is a complete local automaton containing $\mathcal{A}$. 


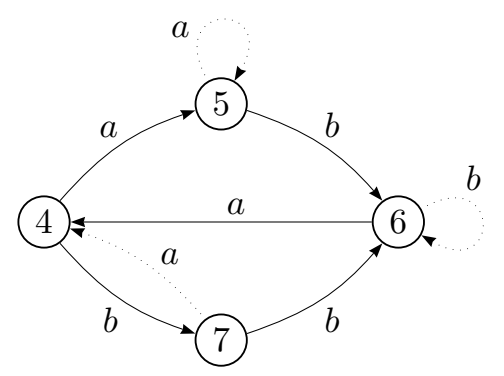

Figure 7: A split $\mathcal{A}_{1}$ of $\mathcal{A}$ and its completion $\mathcal{B}_{1}$ with the dotted edges.

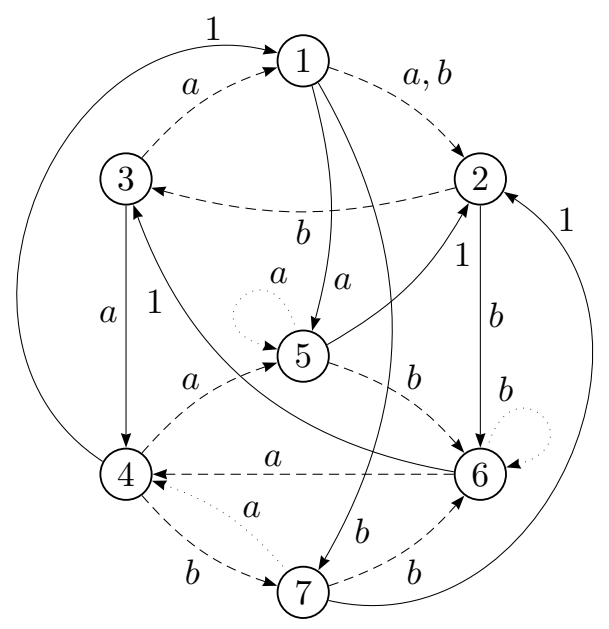

Figure 8: The auxiliary graph (with continuous edges). The automata $\mathcal{A}$ and $\mathcal{A}_{1}$ are represented with dashed edges and the additional edges with dotted lines. 
To check that it is complete, one can apply the subset construction to build an equivalent deterministic automaton. Since $\mathcal{A}$ is irreducible and local, this construction may be started from any state. Then we can check that each state has outgoing edges carrying each letter.

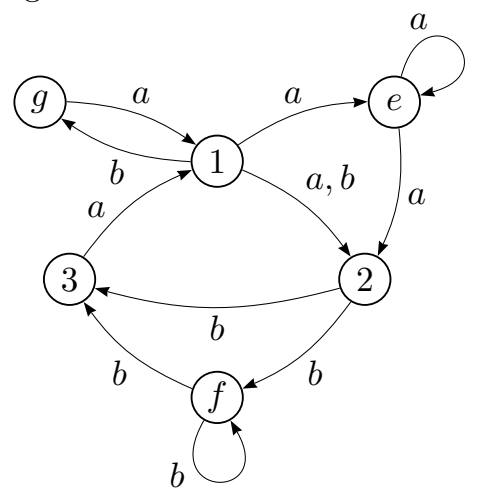

Figure 9: The result is the embedding of $\mathcal{A}$ with respect to $\mathcal{A}_{1}$ and $\mathcal{B}_{1}$. This automaton has order 3 although it is $(3,1)$-local.

Theorem 2 can be generalized as follows. Let $\mathcal{A}$ be a local automaton. Any local automaton $\mathcal{B}$ such that $L(\mathcal{B}) \subseteq L(\mathcal{A})$ is a subautomaton of a local automaton $\mathcal{C}$ strongly equivalent to $\mathcal{A}$. Moreover, if $\mathcal{A}$ is an automaton of order at most one, then the order of $\mathcal{C}$ can be chosen equal to the order of $\mathcal{B}$. Theorem 2 corresponds to the case where the automaton $\mathcal{A}$ has just one state.

Example 7 Let $\mathcal{A}$ be the $(1,0)$-local automaton represented on the right of Figure 10. The automaton $\mathcal{B}$ represented on the left with continuous lines is $(1,1)$-local. One has $L(\mathcal{B}) \subset L(\mathcal{A})$. Adding the dotted edge labelled $a$ to $\mathcal{B}$ gives a $(1,1)$-local automaton $\mathcal{C}$ which is equivalent to the automaton $\mathcal{A}$.
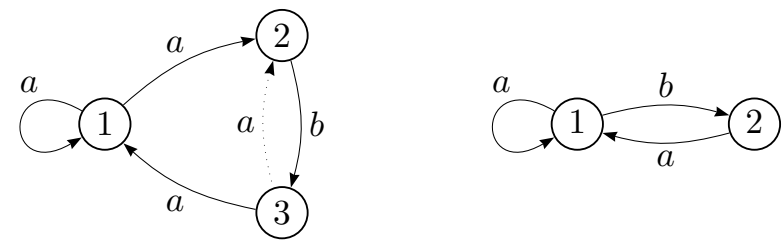

Figure 10: The local automata $\mathcal{B}, \mathcal{C}$ on the left (with the additional dotted edge for $\mathcal{C}$ ), and $\mathcal{A}$ on the right. 


\section{Weakly deterministic automata}

An automaton $\mathcal{A}$ is said to have delay $d$ if for any pair of paths

$$
p \stackrel{a}{\rightarrow} q \stackrel{z}{\rightarrow} r, \quad p \stackrel{a}{\rightarrow} q^{\prime} \stackrel{z}{\rightarrow} r^{\prime},
$$

with $a \in A, z \in A^{*}$ and $|z|=d$ then $q=q^{\prime}$. We say that the automaton is weakly deterministic if it has delay $d$ for some integer $d \geq 0$. The minimal delay of an automaton $\mathcal{A}$ is the least integer $d$ such that $\mathcal{A}$ has delay $d$.

Weakly deterministic automata are also known in symbolic dynamics as right-closing labeled graph.

Thus a deterministic automaton has delay 0. Observe that if $\mathcal{A}$ has delay $d$, then for any word $w$, and for any pair of paths

$$
p \stackrel{w}{\rightarrow} q \stackrel{z}{\rightarrow} r, \quad p \stackrel{w}{\rightarrow} q^{\prime} \stackrel{z}{\rightarrow} r^{\prime},
$$

with $w, z \in A^{*}$ and $|z|=d$, the paths $p \stackrel{w}{\rightarrow} q$ and $p \stackrel{w}{\rightarrow} q^{\prime}$ are equal.

Proposition 11 Let $\mathcal{A}$ be an essential automaton. If $\mathcal{A}$ is local, it is weakly deterministic. If it is weakly deterministic, it is unambiguous.

Proof Suppose first that $\mathcal{A}$ is $(\lambda, \rho)$-local. Let $p \stackrel{a}{\rightarrow} q \stackrel{z}{\rightarrow} r$ and $p \stackrel{a}{\rightarrow} q^{\prime} \stackrel{z}{\rightarrow} r^{\prime}$ be paths with $|z|=\rho$. Since $\mathcal{A}$ is essential, there exists a path $s \stackrel{y}{\rightarrow} p$ with $|y a| \geq \lambda$. Since we have paths $s \stackrel{y a}{\rightarrow} q \stackrel{z}{\rightarrow} r$ and $s \stackrel{y a}{\rightarrow} q^{\prime} \stackrel{z}{\rightarrow} r^{\prime}$, we have $q=q^{\prime}$. Thus $\mathcal{A}$ has delay $\rho$.

Suppose next that $\mathcal{A}$ is has delay $d$. Two paths from $p$ to $q$ with the same label may be extended to the right arbitrarily. This forces them to coincide. Thus $\mathcal{A}$ is unambiguous.

We will use the following result.

Proposition 12 Let $\mathcal{A}_{1}$ and $\mathcal{A}_{2}$ be $(R, S)$-strongly equivalent irreducible automata. If the automata $\mathcal{A}_{1}$ is weakly deterministic, then $\mathcal{A}_{2}$ also, and their minimal delays differ at most by one.

Proof Let us suppose that $\mathcal{A}_{2}$ has delay $d-1$ and show that $\mathcal{A}_{1}$ has delay $d$. We treat the case where the entries of $R$ are in $\mathfrak{P}(A)$ and the entries of $S$ are in $\{0,1\}$. The other case is similar.

Let $p_{0} \stackrel{a_{0}}{\rightarrow} p_{1} \stackrel{a_{1}}{\rightarrow} \cdots \stackrel{p_{d}}{\rightarrow} p_{d+1}$ and $p_{0} \stackrel{a_{0}}{\rightarrow} p_{1}^{\prime} \stackrel{a_{1}}{\rightarrow} \cdots \stackrel{a_{d}}{\rightarrow} p_{d+1}^{\prime}$ be two paths in $\mathcal{A}_{1}$ of length $d+1$ with the same origin and the same label $a_{0} a_{1} \cdots a_{d}$, with $a_{i} \in A$. Since $\mathcal{A}_{1}$ is irreducible, there exists an $S$-edge $q_{0} \rightarrow p_{0}$. Thus we have, in the auxiliary graph, paths $q_{0} \rightarrow p_{0} \stackrel{a_{0}}{\rightarrow} q_{1} \rightarrow p_{1} \stackrel{a_{1}}{\rightarrow} q_{2} \cdots \stackrel{a_{d}}{\rightarrow} p_{d+1}$ and $q_{0} \rightarrow p_{0} \stackrel{a_{0}}{\rightarrow} q_{1}^{\prime} \rightarrow p_{1}^{\prime} \stackrel{a_{1}}{\rightarrow} q_{2}^{\prime} \ldots \stackrel{a_{d}}{\rightarrow} p_{d+1}^{\prime}$. Since $\mathcal{A}_{2}$ has delay $(d-1)$, we have $q_{1}=q_{1}^{\prime}$ and $q_{2}=q_{2}^{\prime}$. This implies $p_{1}=p_{1}^{\prime}$. Thus $\mathcal{A}_{1}$ has delay $d$.

The following statement shows that an input merge cannot increase the delay.

Proposition 13 Let $\mathcal{A}_{1}$ be an input merge of $\mathcal{A}_{2}$. Then $\mathcal{A}_{1}$ and $\mathcal{A}_{2}$ have the same minimal delay. 
Proof The delay of a (input or output) merge cannot be larger than the original one. Hence if $\mathcal{A}_{2}$ has delay $d$, then $\mathcal{A}_{1}$ has delay $d$. Conversely, suppose that $\mathcal{A}_{1}$ has delay $d$. Let $p \stackrel{a}{\rightarrow} q \stackrel{u}{\rightarrow} r$ and $p \stackrel{a}{\rightarrow} q^{\prime} \stackrel{u}{\rightarrow} r^{\prime}$ be two paths in $\mathcal{A}_{2}$ where $a \in A$ and $u \in A^{d}$. We then have paths $\bar{p} \stackrel{a}{\rightarrow} \bar{q} \stackrel{u}{\rightarrow} \bar{r}$ and $\bar{p} \stackrel{a}{\rightarrow} \overline{q^{\prime}} \stackrel{u}{\rightarrow} \overline{r^{\prime}}$ in $\mathcal{A}_{1}$. Since $\mathcal{A}_{1}$ has delay $d$, we have $\bar{q}=\bar{q}^{\prime}$. Since the inputs of $q$ and $q^{\prime}$ are not disjoint, we have $q=q^{\prime}$. Thus $\mathcal{A}_{2}$ has delay $d$.

The following result, which already appears in [1], is the analogue of Theorem 2 for weakly deterministic automata. The proof uses again Nasu embeddings.

Theorem 3 A weakly deterministic irreducible automaton is a subautomaton of a complete one with the same delay.

Observe that, contrary to Theorem 2, the above statement is trivial if the result is not required to be irreducible.

We first prove the following result which shows that a weakly deterministic irreducible automaton is strongly equivalent to a deterministic irreducible automaton.

Proposition 14 If $\mathcal{A}$ is a weakly deterministic irreducible automaton with delay $d \geq 1$, there exist automata $\mathcal{A}_{1}$ and $\mathcal{A}_{2}$ such that $\mathcal{A}$ is an input merge of $\mathcal{A}_{1}, \mathcal{A}_{2}$ is an output merge of $\mathcal{A}_{1}$ and $\mathcal{A}_{2}$ has delay $d-1$.

Proof Set $\mathcal{A}_{0}=\mathcal{A}$ and $\mathcal{A}_{i}=\left(P_{i}, E_{i}\right)$ for $i=0,1,2$. Let $P_{1}=E_{0}$ and let $E_{1}$ be the set of triples $(e, a, f)$ with $e, f \in E_{0}$ and $a \in A$ such that $e, f$ are consecutive edges of $\mathcal{A}$ and $a$ is the label of $f$. In this way, there is an input merge from $\mathcal{A}_{1}$ to $\mathcal{A}_{0}$ obtained by merging the states with the same third component. Let $\mathcal{A}_{2}$ be the automaton obtained from $\mathcal{A}_{1}$ by merging the states having the same input. By Proposition 13 and since $\mathcal{A}_{0}$ has delay $d, \mathcal{A}_{1}$ has delay $d$. Let us verify that $\mathcal{A}_{2}$ has delay $d-1$. Consider two paths $\alpha \stackrel{a}{\rightarrow} \beta \stackrel{u}{\rightarrow} \gamma$ and $\alpha \stackrel{a}{\rightarrow} \beta^{\prime} \stackrel{u}{\rightarrow} \gamma^{\prime}$ in $\mathcal{A}_{2}$ with $a \in A$ and $u \in A^{d-1}$. These paths are the image under the output merge of two paths in $\mathcal{A}_{1}$ of the form $(i, b, p) \stackrel{a}{\rightarrow}(p, a, q) \stackrel{u}{\rightarrow}(r, c, s)$ and $\left(i^{\prime}, b, p^{\prime}\right) \stackrel{a}{\rightarrow}\left(p^{\prime}, a, q^{\prime}\right) \stackrel{u}{\rightarrow}\left(r^{\prime}, c, s^{\prime}\right)$. Since $(i, b, p)$ and $\left(i^{\prime}, b, p^{\prime}\right)$ have the same input in $\mathcal{A}_{1}$, we have $i=i^{\prime}$. Thus we obtain the two paths $i \stackrel{b}{\rightarrow} p \stackrel{a u}{\rightarrow} s$ and $i \stackrel{b}{\rightarrow} p^{\prime} \stackrel{a u}{\rightarrow} s$ in $\mathcal{A}$. Since $\mathcal{A}$ has delay $d$, this forces $p=p^{\prime}$. But since $(p, a, q)$ and $\left(p, a, q^{\prime}\right)$ have the same input in $\mathcal{A}_{1}$, they belong to the same class of the output merge equivalence giving $\mathcal{A}_{2}$. This forces $\beta=\beta^{\prime}$.

Proof of Theorem 3. We use an induction on the minimal delay $d$ of $\mathcal{A}$. If $d=0$, then $\mathcal{A}$ is deterministic and the property is clearly true. Suppose $d \geq 1$. Let $\mathcal{A}_{0}=\mathcal{A}$ and $\mathcal{A}_{1}, \mathcal{A}_{2}$ be the automata given by Proposition 14 . The construction is illustrated in Figure 11. By induction, $\mathcal{A}_{2}$ is a subautomaton of an irreducible automaton $\mathcal{B}_{2}$ with delay $d-1$. Let $\mathcal{B}_{1}$ be the Nasu embedding of $\mathcal{A}_{1}$ with respect to $\left(\mathcal{A}_{2}, \mathcal{B}_{2}\right)$. By Proposition $12, \mathcal{A}_{1}$ has delay $d$. By Proposition 5 , there is an input merge $\mathcal{B}_{0}$ of $\mathcal{B}_{1}$ such that $\mathcal{A}_{0}$ is a subautomaton of $\mathcal{B}_{0}$. By Proposition 13, $\mathcal{B}_{0}$ has delay $d$, which concludes the proof. 


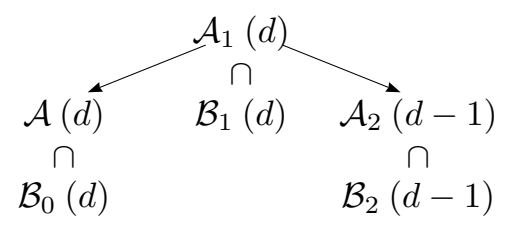

Figure 11: The construction in the proof of Proposition 14. The delay of each automaton is given inside the parenthesis.

Example 8 Figure 12 illustrates the construction of the completion of a weakly deterministic automaton.
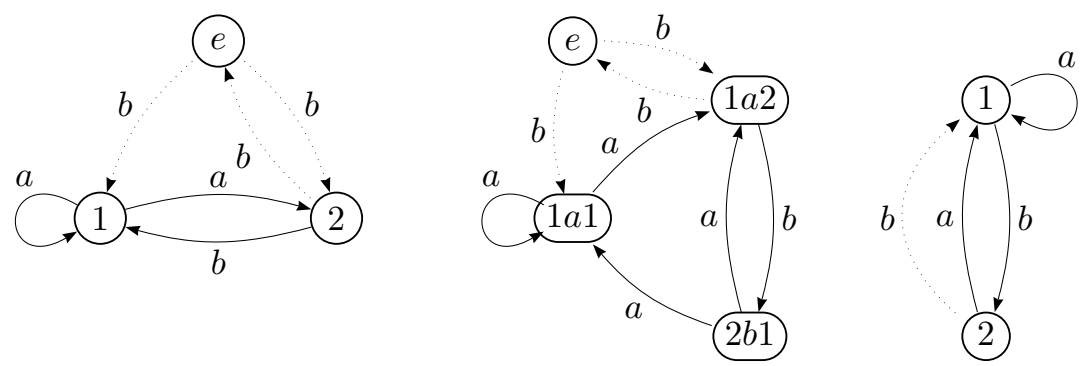

Figure 12: Let $\mathcal{A}=\mathcal{A}_{0}$ be the automaton with delay 1 represented on the left with continuous lines. The automaton $\mathcal{A}_{1}$ is represented at the center. The automaton $\mathcal{A}_{2}$ obtained by merging states $1 a 1$ and $1 a 2$ is shown on the right. It is deterministic and can be completed by adding an edge $e$ labelled $b$ from state 2 to state 1 represented with dashes (another solution would be to add a loop labelled $b$ on 2). Working backwards, the Nasu embeddings of $\mathcal{A}_{1}$ and $\mathcal{A}_{0}$ appear with dashes. The result on the left is a complete automaton $\mathcal{B}_{0}$ with delay 1 containing $\mathcal{A}_{0}$ as a subautomaton.

The particular solution given in [1] is not the same as the one described above. Let us explain how it works on this example.

The embedding is based on the observation that an automaton with delay $d$ is complete if and only if for each state $p$ the following condition is satisfied: for each word $u$ of length $d$ such that there is a path labelled $u$ starting at $p$, there also a path labelled $u a$ starting at $p$ for each $a \in A$.

Since state 2 can be followed by $b a$ but not by $b b$, we add an edge labelled $b$ from 2 to a new state called $b$ and such that there is an edge labelled $b$ going to state 1 . Further, since state $b$ can be followed by $b a$ but not by $b b$, we add a loop labelled $b$ on state $b$. The above condition is now satisfied for any state. Let $\mathcal{B}_{0}$ be the complete automaton thus obtained.

This automaton is obtained by the previous construction starting with the 

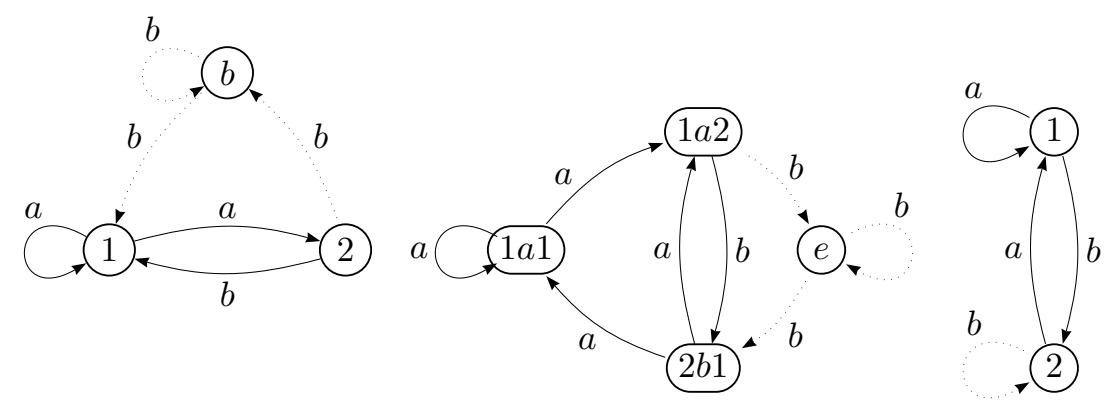

Figure 13: Another embedding of the previous automaton.

completion of the deterministic automaton $\mathcal{A}_{2}$ shown on the right and working backwards as before.

The full statement of the result of [1] is more general and Theorem 3 and its proof can be extended as follows. Let $\mathcal{A}$ be an irreducible edge automaton. Any weakly deterministic irreducible automaton $\mathcal{B}$ such that $L(\mathcal{B}) \subseteq L(\mathcal{A})$ is a subautomaton of a weakly deterministic irreducible automaton $\mathcal{C}$ which is equivalent to $\mathcal{A}$ and has the same delay.

We have not been able to found a way to prove by Nasu extensions the analogous result of [1] concerning bi-closing automata, i.e. which have finite delay in both directions.

Acknowledgements: We would like to thank Brian Marcus who pointed out to us the possibility of using Nasu's masking lemma in this problem.

\section{References}

[1] Jonathan Ashley, Brian Marcus, Dominique Perrin, and Selim Tuncel. Surjective extensions of sliding block codes. SIAM J. Discrete Math., 6:582611, 1993.

[2] Marie-Pierre Béal, Sylvain Lombardy, and Dominique Perrin. Embeddings of local automata. In Information Theory, ISIT 2008, pages 2351-2355. IEEE, 2008.

[3] Jean Berstel, Dominique Perrin, and Christophe Reutenauer. Codes and Automata. Cambridge University Press, 2007. To appear, see http:// www-igm. univ-mlv.fr/ berstel/LivreCodes/newlivreCodes.pdf.

[4] Véronique Bruyère. On maximal codes with bounded synchronization delay. Theor. Comput. Sci., 204:11-28, 1988.

[5] Andrei Ehrenfeucht and Gregorz Rozenberg. Each regular code is included in a regular maximal code. RAIRO Informat. Theor., 20:89-96, 1983. 
[6] Wolfgang Krieger. On sofic systems. I. Israel J. Math., 48(4):305-330, 1984.

[7] Douglas A. Lind and Brian H. Marcus. An Introduction to Symbolic Dynamics and Coding. Cambridge University Press, 1995.

[8] Rosalba Montalbano. Local automata and completion. In STACS 93, volume 665 of Lecture Notes in Comput. Sci., pages 333-342. Springer verlag, 1993.

[9] Masakazu Nasu. Topological conjugacy for sofic systems. Ergodic Theory Dynam. Systems, 6(2):265-280, 1986.

[10] Masakazu Nasu. Topological conjugacy for sofic systems and extensions of automorphisms of finite subsystems of topological markov shifts. In Proceedings of Maryland special year in Dynamics 1986-87, volume 1342 of Lecture Notes in Mathematics, pages 564-607. Springer verlag, 1988.

[11] Marcel-Paul Schützenberger. A remark on incompletely specified automata. Information and Control, 8:373-376, 1965. 